\title{
ASYMPTOTIC BEHAVIOUR FOR WALL POLYNOMIALS AND THE ADDITION FORMULA FOR LITTLE q-LEGENDRE POLYNOMIALS*
}

\author{
WALTER VAN ASSCHE† AND TOM H. KOORNWINDER $\ddagger$
}

Abstract. Wall polynomials $W_{n}(x ; b, q)$ are considered and their asymptotic behaviour is described when $q=c^{1 / n}$ and $n$ tends to infinity. The results are then used to derive the addition and product formulas for the Legendre polynomials from the recently obtained addition and product formulas for little $q$-Legendre polynomials.

Key words. Wall polynomials, addition formula, product formula, basic hypergeometric polynomials, Legendre polynomials

AMS(MOS) subject classifications. 33 A65, 42C05

1. Introduction. The Wall polynomials $W_{n}(x ; b, q)$ are defined by the recurrence formula

$$
\begin{aligned}
W_{n+1}(x ; b, q)=\{ & \left.-\left[b+q-(1+q) b q^{n}\right] q^{n}\right\} W_{n}(x ; b, q) \\
& -b\left(1-q^{n}\right)\left(1-b q^{n-1}\right) q^{2 n} W_{n-1}(x ; b, q), \quad n=0,1,2, \cdots
\end{aligned}
$$

with initial values $W_{-1}=0$ and $W_{0}=1$. Clearly $W_{n}(x ; b, q)$ is a monic polynomial of degree $n$ in the variable $x$. Some properties of Wall polynomials are given in Chihara's book $[4$, p. 198]. These polynomials are closely related to the continued fraction

$$
1+\frac{x}{1}+\frac{(1-b) q x}{1}+\frac{(1-q) b q x}{1}+\frac{(1-b q) q^{2} x}{1}+\cdots
$$

which was studied by H. S. Wall [16]. The Wall polynomials were also studied by Chihara [5] because they have a Brenke-type generating function, i.e.,

$$
\sum_{n=0}^{\infty} W_{n}(x ; b, q) \frac{z^{n}}{(b ; q)_{n}(q ; q)_{n}}=A(z) B(z x),
$$

where

$$
\begin{aligned}
& A(z)=\sum_{n=0}^{\infty}(-1)^{n} q^{n(n+1) / 2} \frac{z^{n}}{(q ; q)_{n}}=(z q ; q)_{\infty}, \\
& B(z)=\sum_{n=0}^{\infty} \frac{z^{n}}{(b ; q)_{n}(q ; q)_{n}} .
\end{aligned}
$$

We have used the notation

$$
\begin{aligned}
& (b ; q)_{n}=(1-b)(1-b q) \cdots\left(1-b q^{n-1}\right) \\
& (b ; q)_{\infty}=\lim _{n \rightarrow \infty}(b ; q)_{n}
\end{aligned}
$$

* Received by the editors March 13, 1989; accepted for publication (in revised form) October $10,1989$.

$\dagger$ Catholic University of Leuven, Department of Mathematics, Celestijnenlaan 200B, B-3030 Leuven, Belgium. This author is a Research Associate of the Belgium National Fund for Scientific Research.

$\ddagger$ Centre for Mathematics and Computer Science, P.O. Box 4079, NL-1009 AB Amsterdam, the Netherlands. 
the latter limit exists whenever $|q|<1$. From this generating function we easily find

$$
\begin{aligned}
W_{n}(x ; b, q) & =(-1)^{n}(b ; q)_{n} q^{n(n+1) / 2} \sum_{k=0}^{n} \frac{(q ; q)_{n}}{(q ; q)_{n-k}(q ; q)_{k}} q^{k(k-1) / 2} \frac{\left(-q^{-n} x\right)^{k}}{(b ; q)_{k}} \\
& =(-1)^{n}(b ; q)_{n} q^{n(n+1) / 2}{ }_{2} \phi_{1}\left(q^{-n}, 0 ; b ; q, x\right),
\end{aligned}
$$

where the $q$-hypergeometric (or basic hypergeometric [6]) function is defined by

$$
{ }_{r+1} \phi_{r}\left(a_{1}, \cdots, a_{r+1} ; b_{1}, \cdots, b_{r} ; q, z\right)=\sum_{k=0}^{\infty} \frac{\left(a_{1} ; q\right)_{k} \cdots\left(a_{r+1} ; q\right)_{k}}{\left(b_{1} ; q\right)_{k} \cdots\left(b_{r} ; q\right)_{k}} \frac{z^{k}}{(q ; q)_{k}} .
$$

If $0<q<1$ and $0<b<1$ then the Wall polynomials are orthogonal with respect to a positive measure supported on the geometric sequence $\left\{q^{n}: n=1,2,3, \cdots\right\}$ and we have

$$
\sum_{k=0}^{\infty} W_{n}\left(q^{k+1} ; b, q\right) W_{m}\left(q^{k+1} ; b, q\right) \frac{b^{k}}{(q ; q)_{k}}=0, \quad n \neq m .
$$

The orthonormal polynomials are given by

$$
w_{n}(x ; b, q)=\frac{q^{-n(n+1) / 2}}{\sqrt{b^{n}(q ; q)_{n}(b ; q)_{n}}} W_{n}(x ; b, q),
$$

and they satisfy

$$
(b ; q)_{\infty} \sum_{k=0}^{\infty} w_{n}\left(q^{k+1} ; b, q\right) w_{m}\left(q^{k+1} ; b, q\right) \frac{b^{k}}{(q ; q)_{k}}=\delta_{n, m}, \quad n, m \geqq 0
$$

and the three-term recurrence relation (1.1) becomes

$$
x w_{n}(x ; b, q)=a_{n+1} w_{n+1}(x ; b, q)+b_{n} w_{n}(x ; b, q)+a_{n} w_{n-1}(x ; b, q)
$$

with $w_{-1}=0, w_{0}=1$, and

$$
\begin{array}{ll}
a_{n}=a_{n}(b, q)=q^{n} \sqrt{b\left(1-q^{n}\right)\left(1-b q^{n-1}\right)}, & n=1,2,3, \cdots, \\
b_{n}=b_{n}(b, q)=q^{n}\left[b+q-(1+q) b q^{n}\right], & n=0,1,2, \cdots .
\end{array}
$$

Sometimes it is convenient to use the notation

$$
(b ; q)_{\infty} \sum_{k=0}^{\infty} f\left(q^{k+1}\right) \frac{b^{k}}{(q ; q)_{k}}=\int_{0}^{1} f(z) d \mu(z ; b, q), \quad f \in C[0,1]
$$

so that $\mu(\cdot ; b, q)$ is the orthogonality measure for the Wall polynomials $W_{n}(x ; b, q)$.

Recently Koornwinder [8] obtained the addition formula for little $q$-Legendre polynomials by using the fact that the matrix elements of the irreducible unitary representations of the quantum group $S_{\mu} U(2)$ (see, e.g., Woronowicz [17], [18]) can be expressed in terms of little $q$-Jacobi polynomials (Masuda et al. [9], Vaksman and Soibelman [13], Koornwinder [7]). The little $q$-Jacobi polynomials are defined in terms of $q$-hypergeometric functions by

$$
p_{n}(x ; a, b \mid q)={ }_{2} \phi_{1}\left(q^{-n}, a b q^{n+1} ; a q ; q, q x\right) .
$$

If $a=q^{\alpha}$ and $b=q^{\beta}$ then these little $q$-Jacobi polynomials approach the Jacobi polynomials $P_{n}^{(\alpha, \beta)}(1-2 x) / P_{n}^{(\alpha, \beta)}(1)$ as $q$ tends to 1 [1], [3]. If $a=b=1$ then we have 
the little $q$-Legendre polynomials. Notice that for $b=0$ we essentially have the Wall polynomials:

$$
\begin{aligned}
p_{n}\left(\frac{x}{q} ; \frac{b}{q}, 0 \mid q\right) & =(-1)^{n} \frac{q^{-n(n+1) / 2}}{(b ; q)_{n}} W_{n}(x ; b, q) \\
& =(-1)^{n}\left\{\frac{b^{n}(q ; q)_{n}}{(b ; q)_{n}}\right\}^{1 / 2} w_{n}(x ; b, q)
\end{aligned}
$$

The addition formula for little $q$-Legendre polynomials is

$$
\begin{aligned}
& p_{m}\left(q^{z} ; 1,1 \mid q\right) p_{y}\left(q^{z} ; q^{x}, 0 \mid q\right) \\
& =p_{m}\left(q^{x+y} ; 1,1 \mid q\right) p_{m}\left(q^{y} ; 1,1 \mid q\right) p_{y}\left(q^{z} ; q^{x}, 0 \mid q\right) \\
& \quad+\sum_{k=1}^{m} \frac{(q ; q)_{x+y+k}(q ; q)_{m+k} q^{k(y-m+k)}}{(q ; q)_{x+y}(q ; q)_{m-k}(q ; q)_{k}^{2}} p_{m-k}\left(q^{x+y} ; q^{k}, q^{k} \mid q\right) \\
& \quad \cdot p_{m-k}\left(q^{y} ; q^{k}, q^{k} \mid q\right) p_{y+k}\left(q^{z} ; q^{x}, 0 \mid q\right) \\
& \quad+\sum_{k=1}^{m} \frac{(q ; q)_{y}(q ; q)_{m+k} q^{k(x+y-m+1)}}{(q ; q)_{y-k}(q ; q)_{m-k}(q ; q)_{k}^{2}} p_{m-k}\left(q^{x+y-k} ; q^{k}, q^{k} \mid q\right) \\
& \quad \cdot p_{m-k}\left(q^{y-k} ; q^{k}, q^{k} \mid q\right) p_{y-k}\left(q^{z} ; q^{x}, 0 \mid q\right)
\end{aligned}
$$

with $x, y, z=0,1,2, \cdots$. Rahman [11] has given an analytic proof of this addition formula while Rahman and Verma [12] have given similar formulas for the continuous $q$-ultraspherical polynomials. The right-hand side of the above formula can be considered as an expansion of the left-hand side in terms of Wall polynomials. For $q \uparrow 1$ we should get the familiar addition formula for Legendre polynomials (see, e.g., [2, pp. 29-38]), but this limit involves some interesting asymptotic formulas for the Wall polynomials $W_{n}\left(x ; b, c^{1 / n}\right)$ with $0<c<1$ and $n$ tending to infinity. This was the main reason for investigating such asymptotic formulas for Wall polynomials.

In $\S 2$ we establish some weak asymptotics for Wall polynomials. In $\S 3$ we show how the addition formula for Legendre polynomials can be obtained from the addition formula for little $q$-Legendre polynomials by letting $q \rightarrow 1$, and in $\S 4$ we obtain the familiar product formulas for Legendre polynomials from the product formulas for little $q$-Legendre polynomials.

2. Weak asymptotics for Wall polynomials. For little $q$-Jacobi polynomials $p_{n}(x ; a, b \mid q)$ we can put $a=q^{\alpha}$ and $b=q^{\beta}$ and let $q \uparrow 1$ to find Jacobi polynomials on $[0,1]$. However, if either $a$ or $b$ is zero, which is exactly what happens for Wall polynomials, then the limit as $q \uparrow 1$ is $\left(1+(x /(a-1))^{n}\right.$. Therefore another approach is needed to handle the behaviour of Wall polynomials as $q \uparrow 1$. It turns out that we can find some relevant results if we consider the polynomials $W_{n}\left(x ; b, c^{1 / n}\right)$ for $n \rightarrow \infty$. We will prove a more general result for orthonormal polynomials $\left\{p_{k}(x ; n): k=0,1,2, \cdots\right.$; $n \in \mathbb{N}\}$, where $k$ is the degree of the polynomial and $n$ an extra (discrete) parameter. The recurrence formula for these polynomials is given by

$$
x p_{k}(x ; n)=a_{k+1, n} p_{k+1}(x ; n)+b_{k, n} p_{k}(x ; n)+a_{k, n} p_{k-1}(x ; n),
$$

where $a_{k, n}>0, b_{k, n} \in \mathbb{R}, p_{0}(x ; n)=1$, and $p_{-1}(x ; n)=0$. Orthogonal polynomials with regularly varying recurrence coefficients [15] are of this type. 
THEOREM 1. Assume that $[r, s]$ is a finite interval that, for all $n$, contains the support of the orthogonality measure for $\left\{p_{k}(x ; n)\right\}$. Assume moreover that

$$
\lim _{n \rightarrow \infty} a_{n, n}=A>0, \quad \lim _{n \rightarrow \infty} b_{n, n}=B \in \mathbb{R}
$$

and that

$$
\lim _{n \rightarrow \infty}\left(a_{k, n}^{2}-a_{k-1, n}^{2}\right)=0, \quad \lim _{n \rightarrow \infty}\left(b_{k, n}-b_{k-1, n}\right)=0,
$$

uniformly in $k$, then

$$
\lim _{n \rightarrow \infty} \frac{p_{n+1}(x ; n)}{p_{n}(x ; n)}=\rho\left(\frac{x-B}{2 A}\right),
$$

uniformly on compact sets of $\mathbb{C} \backslash[r, s]$, where $\rho(x)=x+\sqrt{x^{2}-1}$ (the square root here is defined to be the one for which $|\rho(x)|>1$ for $x \in \mathbb{C} \backslash[-1,1])$.

Proof. Let $K$ be a compact set in $\mathbb{C} \backslash[r, s]$; then the distance between $K$ and $[r, s]$ is strictly positive. Denote this distance by $\delta>0$. A decomposition into partial fractions gives

$$
\frac{p_{k-1}(x ; n)}{p_{k}(x ; n)}=a_{k, n} \sum_{j=1}^{k} \frac{d_{j, k}}{x-x_{j, k}},
$$

where $\left\{x_{j, k}: 1 \leqq j \leqq k\right\}$ are the zeros of $p_{k}(x ; n)$ and $\left\{d_{j, k}: 1 \leqq j \leqq k\right\}$ are positive numbers adding up to 1 . Since all the zeros of $p_{k}(x ; n)$ are in $[r, s]$ we have $\left|x-x_{j, k}\right|>\delta$ for $x \in K$ and therefore

$$
\left|\frac{p_{k-1}(x ; n)}{p_{k}(x, n)}\right|<\frac{a_{k, n}}{\delta}
$$

holds uniformly for $x \in K$. Consider the Turán determinant

$$
D_{k}(x ; n)=p_{k}^{2}(x ; n)-\frac{a_{k+1, n}}{a_{k, n}} p_{k+1}(x ; n) p_{k-1}(x ; n) .
$$

By using the recurrence relation (2.1) we find

$$
\begin{gathered}
D_{k}(x ; n)=D_{k-1}(x ; n)+\frac{b_{k, n}-b_{k-1, n}}{a_{k, n}} p_{k}(x ; n) p_{k-1}(x ; n) \\
+\frac{a_{k, n}^{2}-a_{k-1, n}^{2}}{a_{k, n} a_{k-1, n}} p_{k-2}(x ; n) p_{k}(x ; n)
\end{gathered}
$$

(see $[14$, Thm. 4.10 , p. 117]). If we define

$$
R_{k, n}(x)=\frac{D_{k}(x ; n)}{p_{k+1}(x ; n) p_{k}(x ; n)},
$$

then by (2.6)

$$
\begin{gathered}
\left|R_{k, n}(x)\right| \leqq\left|R_{k-1, n}(x)\right| \frac{p_{k-1}(x ; n)}{p_{k+1}(x ; n)}\left|+\frac{\left|b_{k, n}-b_{k-1, n}\right|}{a_{k, n}}\right| \frac{p_{k-1}(x ; n)}{p_{k+1}(x ; n)} \mid \\
+\frac{\left|a_{k, n}^{2}-a_{k-1, n}^{2}\right|}{a_{k, n} a_{k-1, n}}\left|\frac{p_{k-2}(x ; n)}{p_{k+1}(x ; n)}\right|,
\end{gathered}
$$

so that by (2.5) we have for $x \in K$

$$
\left|R_{k, n}(x)\right| \leqq \frac{a_{k, n} a_{k+1, n}}{\delta^{2}}\left|R_{k-1, n}(x)\right|+\left|b_{k, n}-b_{k-1, n}\right| \frac{a_{k+1, n}}{\delta^{2}}+\left|a_{k, n}^{2}-a_{k-1, n}^{2}\right| \frac{a_{k+1, n}}{\delta^{3}} .
$$


By the conditions imposed there exists a constant $C$ such that $a_{k, n}<C$ for every $n$ and $k$ (cf. [4, Chap. IV, Example 2.12]). Therefore, by (2.3),

$$
\left|R_{k, n}(x)\right| \leqq\left(\frac{C}{\delta}\right)^{2}\left|R_{k-1, n}(x)\right|+A_{n}, \quad x \in K
$$

where $A_{n} \rightarrow 0$ as $n \rightarrow \infty$. Iteration gives

$$
\left|R_{n, n}(x)\right| \leqq A_{n} \frac{(C / \delta)^{2 n}-1}{(C / \delta)^{2}-1}+\left|R_{0, n}(x)\right|(C / \delta)^{2 n}, \quad x \in K .
$$

If $\delta>C$ then obviously $R_{n, n}(x) \rightarrow 0$ as $n \rightarrow \infty$ (use $\left|R_{0, n}\right|=\left|p_{0}(x ; n) / p_{1}(x ; n)\right|<a_{1, n} / \delta$ ), which by (2.2), (2.3), and (2.5) leads to

$$
\lim _{n \rightarrow \infty}\left|\frac{p_{n}(x ; n)}{p_{n+1}(x ; n)}-\frac{p_{n-1}(x ; n)}{p_{n}(x ; n)}\right|=0,
$$

uniformly for $x \in K$ (provided $\delta>C$ ). By (2.5) the sequence of analytic functions $p_{n}(x ; n) / p_{n+1}(x ; n)$ is uniformly bounded on compact sets of $\mathbb{C} \backslash[r, s]$ and thus there exists a subsequence converging to some function $L(x)$, uniformly on $K$. Use the recurrence formula (2.1) and the properties (2.2), (2.3), and (2.7) to find that this limit satisfies

$$
x=\frac{A}{L(x)}+B+A L(x)
$$

and since $\left|p_{n}(x ; n) / p_{n+1}(x ; n)\right|<C / \delta<1$ for $x \in K$ by (2.5) we have

$$
\frac{1}{L(x)}=\rho\left(\frac{x-B}{2 A}\right)
$$

This gives the result for $\delta>C$. This can be extended to hold for $\delta>0$ by using the Stieltjes-Vitali theorem (cf. [4, p. 121]) and the uniform bound (2.5).

Remark. The asymptotic behaviour actually holds uniformly on compact sets of $\mathbb{C} \backslash \Omega$, where $\Omega$ is the closure of the set of zeros of $p_{n}(x ; n)$ as $n$ runs through the integers. Clearly, $\Omega$ is a subset of $[r, s]$ since the zeros of $p_{n}(x ; n)$ are all inside the interval $[r, s]$. The condition that the joint supports of the orthogonality measures should be contained in the finite interval $[r, s]$ can also be relaxed. Only the zeros of $p_{k}(x ; n)(k \leqq n+1, n=0,1,2, \cdots)$ must lie in $[r, s]$.

Corollary 1. Suppose $0<b<1$ and $0<c<1$. Then

$$
\lim _{n \rightarrow \infty} \frac{W_{n+k}\left(x ; b, c^{1 / n}\right)}{W_{n}\left(x ; b, c^{1 / n}\right)}=\left\{b(1-c)(1-b c) c^{2}\right\}^{k / 2} \rho^{k}\left(\frac{x-[b+1-2 b c] c}{2 c \sqrt{b(1-c)(1-b c)}}\right)
$$

uniformly on compact sets of $\mathbb{C} \backslash[0,1]$.

Proof. The proof follows immediately from

$$
\lim _{n \rightarrow \infty} \frac{W_{n+k}\left(x ; b, c^{1 / n}\right)}{W_{n+k-1}\left(x ; b, c^{1 / n}\right)}-\left\{b(1-c)(1-b c) c^{2}\right\}^{1 / 2} \rho\left(\frac{x-[b+1-2 b c] c}{2 c \sqrt{b(1-c)(1-b c)}}\right),
$$

which in turn can be proved by using Theorem 1 with recurrence coefficients $a_{k, n}=$ $a_{k}\left(b, c^{1 / n}\right)$ and $b_{k, n}=b_{k}\left(b, c^{1 / n}\right)$ given by (1.6).

Corollary 2. Suppose $0<b<1$ and $0<c<1$. Then

$$
\lim _{n \rightarrow \infty} \frac{p_{n+k}\left(z ; b, 0 \mid c^{1 / n}\right)}{p_{n}\left(z ; b, 0 \mid c^{1 / n}\right)}=(-1)^{k}\left\{\frac{b(1-c)}{1-b c}\right\}^{k / 2} \rho^{k}\left(\frac{z-[b+1-2 b c] c}{2 c \sqrt{b(1-c)(1-b c)}}\right)
$$


uniformly for $z$ on compact subsets of $\mathbb{C} \backslash[0,1]$, where $p_{n}(x ; a, b \mid q)$ are the little $q$-Jacobi polynomials.

Proof. This follows immediately from (1.8) and Corollary 1.

It is important in the asymptotic formula (2.4) that the variable $x$ stays away from the zeros of $p_{n}(x ; n)$. On the set $\Omega$, the closure of the zeros of $p_{n}(x ; n)$, the orthogonal polynomials will oscillate. The following theorem gives a result about the weak convergence of measures involving the polynomials $p_{k}(x ; n)$ on $[r, s]$ in terms of their orthogonality measures.

THEOREM 2. Assume that $[r, s]$ is a finite interval that, for all $n$, contains the support of the orthogonality measure $\mu_{n}$ for the orthonormal polynomials $\left\{p_{k}(x ; n): k=\right.$ $0,1,2, \cdots\}$. Assume, moreover, that for all $k \in \mathbb{Z}$

$$
\lim _{n \rightarrow \infty} a_{n+k, n}=A, \quad \lim _{n \rightarrow \infty} b_{n+k, n}=B ;
$$

then for every continuous function $f$ on $[r, s]$

$$
\lim _{n \rightarrow \infty} \int_{r}^{s} f(z) p_{n}(z ; n) p_{n+k}(z ; n) d \mu_{n}(z)=\frac{1}{\pi} \int_{B-2 A}^{B+2 A} \frac{f(z) T_{k}((z-B) /(2 A))}{\sqrt{4 A^{2}-(z-B)^{2}}} d z,
$$

where $T_{n}(x)$ are the Chebyshev polynomials of the first kind.

Proof. We follow the ideas of Nevai and Dehesa [10, Lemma 3]. Let $m$ be a positive integer and apply the recurrence formula (2.1) repeatedly to get

$$
z^{m} p_{n}(z ; n)=\sum_{\substack{-1 \leq k_{i} \leq 1 \\ i=1,2, \cdots, m}} \alpha_{n, n+k_{1}} \alpha_{n+k_{1}, n+k_{1}+k_{2}} \cdots \alpha_{n+k_{1}+\cdots+k_{m-1}, n+k_{1}+\cdots+k_{m}} p_{n+k_{1}+\cdots+k_{m}}(z ; n),
$$

where

$$
\alpha_{j, k}= \begin{cases}a_{j, n} & \text { if } k=j-1 \\ b_{j, n} & \text { if } k=j \\ a_{j+1, n} & \text { if } k=j+1\end{cases}
$$

Hence

$$
\int_{r}^{s} z^{m} p_{n}(z ; n) p_{n+k}(z ; n) d \mu_{n}(z)=\sum_{\substack{-1 \leq k_{i} \leq 1 \\ i=1,2, \cdots, m \\ k_{1}+\cdots+k_{m}=k}} \alpha_{n, n+k_{1}} \alpha_{n+k_{1}, n+k_{1}+k_{2}} \cdots \alpha_{n+k_{1}+\cdots+k_{m-1}, n+k} .
$$

Because of this equation and by (2.10) it follows that the limit as $n \rightarrow \infty$ of $\int_{r}^{s} z^{m} p_{n}(z ; n) p_{n+k}(z ; n) d \mu_{n}(z)$ is the same as the limit of

$$
\frac{1}{2 A^{2} \pi} \int_{B-2 A}^{B+2 A} z^{m} U_{n}\left(\frac{z-B}{2 A}\right) U_{n+k}\left(\frac{z-B}{2 A}\right) \sqrt{4 A^{2}-(z-B)^{2}} d z
$$

since the Chebyshev polynomials of the second kind $U_{n}((z-B) / 2 A)$ are the orthogonal polynomials with constant recurrence coefficients $a_{n}=A$ and $b_{n}=B$. Use the identity

$$
U_{n}(x) U_{n+k}(x)=\frac{1}{2} \frac{T_{k}(x)-T_{2 n+k+2}(x)}{1-x^{2}}
$$

to find

$$
\begin{aligned}
& \frac{1}{2 A^{2} \pi} \int_{B-2 A}^{B+2 A} z^{m} U_{n}\left(\frac{z-B}{2 A}\right) U_{n+k}\left(\frac{z-B}{2 A}\right) \sqrt{4 A^{2}-(z-B)^{2}} d z \\
& \quad=\frac{1}{\pi} \int_{B-2 A}^{B+2 A} \frac{z^{m} T_{k}((z-B) / 2 A)}{\sqrt{4 A^{2}-(z-B)^{2}}} d z-\frac{1}{\pi} \int_{B-2 A}^{B+2 A} \frac{z^{m} T_{2 n+k+2}((z-B) / 2 A)}{\sqrt{4 A^{2}-(z-B)^{2}}} d z .
\end{aligned}
$$


If $2 n+k+2>m$ then the second term on the right-hand side vanishes because of orthogonality, and thus we have the result when $f(x)=x^{m}$. The general result follows from the Hahn-Banach theorem: let the operators $L_{k, n}(k, n=0,1,2, \cdots)$, defined on the Banach space $C[r, s]$ of continuous functions equipped with the supremum norm, be given by

$$
L_{k, n} f=\int_{r}^{s} f(z) p_{n}(z ; n) p_{n+k}(z ; n) d \mu_{n}(z) .
$$

These are uniformly bounded operators because, by Schwarz's inequality and the orthonormality,

$$
\begin{aligned}
& \left|\int_{r}^{s} f(z) p_{n}(z ; n) p_{n+k}(z ; n) d \mu_{n}(z)\right|^{2} \\
& \quad \leqq \int_{r}^{s}|f(z)| p_{n}^{2}(z ; n) d \mu_{n}(z) \int_{r}^{s}|f(z)| p_{n+k}^{2}(z ; n) d \mu_{n}(z) \\
& \quad \leqq\|f\|_{\infty}^{2} .
\end{aligned}
$$

Now use Weierstrass's result that the polynomials form a dense subspace of $C[r, s]$.

COROllary 3. Suppose $0<b<1$ and $0<c<1$. Then for every continuous function $f$ on $[0,1]$

$$
\begin{gathered}
\lim _{n \rightarrow \infty} \int_{0}^{1} f(z) w_{n}\left(z ; b, c^{1 / n}\right) w_{n+k}\left(z ; b, c^{1 / n}\right) d \mu\left(z ; b, c^{1 / n}\right) \\
\quad=\frac{1}{\pi} \int_{B-2 A}^{B+2 A} \frac{f(z) T_{k}((z-B) /(2 A)}{\sqrt{4 A^{2}-(z-B)^{2}}} d z
\end{gathered}
$$

where $A=c \sqrt{b(1-c)(1-b c)}, B=(b+1-2 b c) c$, and $T_{n}(x)$ are the Chebyshev polynomials of the first kind.

Proof. The proof follows because the Wall polynomials $w_{n}\left(x ; b, c^{1 / n}\right)$ satisfy the conditions of Theorem 2, with recurrence coefficients $a_{k, n}=a_{k}\left(b, c^{1 / n}\right)$ and $b_{k}\left(b, c^{1 / n}\right)$ given by (1.6).

3. The addition formula. The little $q$-Legendre polynomials $p_{n}(z ; 1,1 \mid q)$ and the Wall polynomials $p_{n}(z ; a, 0 \mid q)$ are analytic functions of $z$ and the addition formula (1.9) holds for every $z \in\left\{q^{n}: n=0,1,2, \cdots\right\}$ (which is a set with an accumulation point). Therefore it follows that

$$
\begin{aligned}
& p_{m}(z ; 1,1 \mid q) p_{y}\left(z ; q^{x}, 0 \mid q\right) \\
& =p_{m}\left(q^{x+y} ; 1,1 \mid q\right) p_{m}\left(q^{y} ; 1,1 \mid q\right) p_{y}\left(z ; q^{x}, 0 \mid q\right) \\
& \quad+\sum_{k=1}^{m} \frac{(q ; q)_{x+y+k}(q ; q)_{m+k} q^{k(y-m+k)}}{(q ; q)_{x+y}(q ; q)_{m-k}(q ; q)_{k}^{2}} p_{m-k}\left(q^{x+y} ; q^{k}, q^{k} \mid q\right) \\
& \quad \cdot p_{m-k}\left(q^{y} ; q^{k}, q^{k} \mid q\right) p_{y+k}\left(z ; q^{x}, 0 \mid q\right) \\
& \quad+\sum_{k=1}^{m} \frac{(q ; q)_{y}(q ; q)_{m+k} q^{k(x+y-m+1)}}{(q ; q)_{y-k}(q ; q)_{m-k}(q ; q)_{k}^{2}} p_{m-k}\left(q^{x+y-k} ; q^{k}, q^{k} \mid q\right) \\
& \quad \cdot p_{m-k}\left(q^{y-k} ; q^{k}, q^{k} \mid q\right)_{y-k}\left(z ; q^{x}, 0 \mid q\right)
\end{aligned}
$$

holds for every $z \in \mathbb{C}$ and $x, y=0,1,2, \cdots$. It is well known that

$$
\lim _{q \uparrow 1} p_{n}\left(z ; q^{\alpha}, q^{\beta} \mid q\right)=R_{n}^{(\alpha, \beta)}(1-2 z),
$$


where $R_{n}^{(\alpha, \beta)}(x)$ are Jacobi polynomials with the normalization $R_{n}^{(\alpha, \beta)}(1)=1$, i.e., $R_{n}^{(\alpha, \beta)}(x)=P_{n}^{(\alpha, \beta)}(x) / P_{n}^{(\alpha, \beta)}(1)$. Fix $b, c$ in $(0,1)$ such that $\log b / \log c=\beta / \gamma$ with $\beta, \gamma$ positive integers, substitute in (3.1) $q=b^{1 /(n \beta)}=c^{1 /(n \gamma)}, x=n \beta, y=n \gamma$, and let $n \rightarrow \infty$ through the integers. Then by (2.9), (3.1), and (3.2)

$$
\begin{aligned}
& R_{m}^{(0,0)}(1-2 z)=R_{m}^{(0,0)}(1-2 b c) R_{m}^{(0,0)}(1-2 b) \\
&+\sum_{k=1}^{m} \frac{(m+k) !}{(m-k) !(k !)^{2}}(1-b c)^{k} c^{k} R_{m-k}^{(k, k)}(1-2 b c) R_{m-k}^{(k, k)}(1-2 c) \\
& \cdot(-1)^{k}\left\{\frac{b(1-c)}{1-b c}\right\}^{k / 2} \rho^{k}\left(\frac{z-[b+1-2 b c] c}{2 c \sqrt{b(1-c)(1-b c)}}\right) \\
&+\sum_{k=1}^{m} \frac{(m+k) !}{(m-k) !(k !)^{2}}(1-c)^{k}(b c)^{k} R_{m-k}^{(k, k)}(1-2 b c) R_{m-k}^{(k, k)}(1-2 c) \\
& \cdot(-1)^{k}\left\{\frac{1-b c}{b(1-c)}\right\}^{k / 2} \rho^{-k}\left(\frac{z-[b+1-2 b c] c}{2 c \sqrt{b(1-c)(1-b c)}}\right) .
\end{aligned}
$$

Now use the formula $T_{k}(x)=\left[\rho^{k}(x)+\rho^{-k}(x)\right] / 2$; then

$$
\begin{aligned}
R_{m}^{(0,0)}(1-2 z)= & R_{m}^{(0,0)}(1-2 b c) R_{m}^{(0,0)}(1-2 b) \\
& +2 \sum_{k=1}^{m}(-1)^{k} \frac{(m+k) !}{(m-k) !(k !)^{2}} c^{k}[b(1-c)(1-b c)]^{k / 2} \\
& \cdot R_{m-k}^{(k, k)}(1-2 b c) R_{m-k}^{(k, k)}(1-2 c) T_{k}\left(\frac{z-[b+1-2 b c] c}{2 c \sqrt{b(1-c)(1-b c)}}\right) .
\end{aligned}
$$

Finally, choose

$$
\begin{aligned}
& 1-2 z=x y-\sqrt{1-x^{2}} \sqrt{1-y^{2}} t \\
& 1-2 b c=x \\
& 1-2 c=y
\end{aligned}
$$

then

$$
\begin{gathered}
R_{m}^{(0,0)}\left(x y-\sqrt{1-x^{2}} \sqrt{1-y^{2}} t\right)=R_{m}^{(0,0)}(x) R_{m}^{(0,0)}(y) \\
+2 \sum_{k=1}^{m}(-1)^{k} \frac{(m+k) !}{(m-k) !(k !)^{2}} 2^{-2 k}\left\{\sqrt{1-x^{2}} \sqrt{1-y^{2}}\right\}^{k} \\
\cdot R_{m-k}^{(k, k)}(x) R_{m-k}^{(k, k)}(y) T_{k}(t),
\end{gathered}
$$

which is the familiar addition formula for Legendre polynomials. By our method of proof this formula only holds for $t \in \mathbb{C} \backslash \mathbb{R}$ (because we use Corollary 2), but since all the functions considered are analytic in $t$, the result definitely holds for every $t \in \mathbb{C}$.

4. Product formulas. If we multiply both sides of the addition formula (1.9) by $p_{y+k}\left(q^{z} ; q^{x}, 0 \mid q\right) q^{(x+1) z} /(q ; q)_{z}$ and sum from $z=0$ to $\infty$, then by the orthogonality (1.4) and by (1.8)

$$
\begin{aligned}
\sum_{z=0}^{\infty} p_{m} & \left(q^{z} ; 1,1 \mid q\right) p_{y}\left(q^{z} ; q^{x}, 0 \mid q\right) p_{y+k}\left(q^{z} ; q^{x}, 0 \mid q\right) \frac{q^{(x+1) z}}{(q ; q)_{z}} \\
= & \frac{(q ; q)_{x+y+k}(q ; q)_{m+k} q^{k(y-m+k)}}{(q ; q)_{x+y}(q ; q)_{m-k}(q ; q)_{k}^{2}} p_{m-k}\left(q^{x+y} ; q^{k}, q^{k} \mid q\right) p_{m-k}\left(q^{y} ; q^{k}, q^{k} \mid q\right) \\
& \cdot \sum_{z=0}^{\infty} p_{y+k}^{2}\left(q^{z} ; q^{x}, 0 \mid q\right) \frac{q^{(x+1) z}}{(q ; q)_{z}}
\end{aligned}
$$


which holds whenever $k \in\{0,1, \cdots, m\}$. In terms of orthonormal Wall polynomials we have by $(1.8)$

$$
\begin{aligned}
p_{m-k}( & \left.q^{x+y} ; q^{k}, q^{k} \mid q\right) p_{m-k}\left(q^{y} ; q^{k}, q^{k} \mid q\right) \\
= & (-1)^{k} \frac{(q ; q)_{m-k}(q ; q)_{k}^{2}}{(q ; q)_{m+k}} q^{-k(y+k-m)}\left\{q^{-k(x+1)} \frac{(q ; q)_{y}(q ; q)_{x+y}}{(q ; q)_{y+k}(q ; q)_{x+y+k}}\right\}^{1 / 2} \\
& \cdot\left(q^{x+1} ; q\right)_{\infty} \sum_{z=0}^{\infty} p_{m}\left(q^{z} ; 1,1 \mid q\right) w_{y}\left(q^{z+1} ; q^{x+1}, q\right) \\
& \cdot w_{y+k}\left(q^{z+1} ; q^{x+1}, q\right) \frac{q^{(x+1) z}}{(q ; q)_{z}},
\end{aligned}
$$

which can be considered as a product formula for the little $q$-Legendre polynomials and which (for $k=0$ ) is equivalent with the product formula given by Koornwinder [8]. If we use the notation (1.7) then

$$
\begin{gathered}
\left(q^{x+1} ; q\right)_{\infty} \sum_{z=0}^{\infty} p_{m}\left(q^{z} ; 1,1 \mid q\right) w_{y}\left(q^{z+1} ; q^{x+1}, q\right) w_{y+k}\left(q^{z+1} ; q^{x+1}, q\right) \frac{q^{(x+1) z}}{(q ; q)_{z}} \\
=\int_{0}^{1} p_{m}\left(\frac{z}{q} ; 1,1 \mid q\right) w_{y}\left(z ; q^{x+1}, q\right) w_{y+k}\left(z ; q^{x+1}, q\right) d \mu\left(z ; q^{x+1}, q\right) .
\end{gathered}
$$

Fix $b, c$ in $(0,1)$ such that $\log b / \log c=\beta / \gamma$ with $\beta$ and $\gamma$ positive integers and let $q=b^{1 /(n \beta)}=c^{1 /(n \gamma)}, 1+x=n \beta, y=n \gamma$. Then as $n \rightarrow \infty$ we have by Corollary 3 and by the uniform convergence in (3.2) (keep in mind that $p_{m}((z / q) ; 1,1 \mid q)$ is a polynomial of degree $m$ )

$$
\begin{array}{r}
R_{m-k}^{(k, k)}(1-2 b c) R_{m-k}^{(k, k)}(1-2 c)=(-1)^{k} \frac{(m-k) !(k !)^{2}}{(m+k) !} c^{-k}\{b(1-c)(1-b c)\}^{-k / 2} \\
\cdot \frac{1}{\pi} \int_{B-2 A}^{B+2 A} R_{m}^{(0,0)}(1-2 z) \frac{T_{k}((z-B) / 2 A)}{\sqrt{4 A^{2}-(z-B)^{2}}} d z,
\end{array}
$$

where $A=c \sqrt{b(1-c)(1-b c)}$ and $B=(b+1-2 b c) c$. Setting $b c=x, c=y$ gives the familiar product formulas for Legendre polynomials:

$$
\begin{aligned}
R_{m-k}^{(k, k)}(1-2 x) R_{m-k}^{(k, k)}(1-2 y)=(-1)^{k} & \frac{(m-k) !(k !)^{2}}{(m+k) !}\{x y(1-y)(1-x)\}^{-k / 2} \\
& \cdot \frac{1}{\pi} \int_{B-2 A}^{B+2 A} R_{m}^{(0,0)}(1-2 z) \frac{T_{k}((z-B) / 2 A)}{\sqrt{4 A^{2}-(z-B)^{2}}} d z,
\end{aligned}
$$

with $A=\sqrt{x y(1-x)(1-y)}$ and $B=x+y-2 x y$.

\section{REFERENCES}

[1] G. ANDREWS AND R. ASKEY, Enumeration of partitions: The role of Eulerian series and q-orthogonal polynomials, in Higher Combinatorics, M. Aigner, ed., D. Reidel, Dordrecht, the Netherlands, 1977, pp. 3-26.

[2] R. Askey, Orthogonal Polynomials and Special Functions, CBMS-NSF Regional Conference Series in Applied Mathematics 21, Society for Industrial and Applied Mathematics, Philadelphia, 1975.

[3] R. Askey AND J. Wilson, Some Basic Hypergeometric Orthogonal Polynomials that Generalize Jacobi Polynomials, Mem. Amer. Math. Soc. 319, Providence, RI, 1985.

[4] T. S. Chimara, An Introduction to Orthogonal Polynomials, Gordon and Breach, New York, 1978.

[5] - Orthogonal polynomials with Brenke type generating functions, Duke Math. J., 35 (1968), pp. 505-518. 
[6] G. Gasper and M. Rahman, Basic Hypergeometric Series, in Encyclopedia of Mathematics and Its Applications, Vol. 35, Cambridge University Press, Cambridge, 1990.

[7] T. H. KOORNWINDER, Representations of the twisted SU(2) quantum group and some q-hypergeometric orthogonal polynomials, Indag. Math., 51 (1989), pp. 97-117.

[8] - The addition formula for little q-Legendre polynomials and the SU(2) quantum group, SIAM J. Math. Anal., this issue (1991), pp. 292-301.

[9] T. Masuda, K. Mimachi, Y. Nakagami, M. Noumi and K. Ueno, Representations of quantum groups and a q-analogue of orthogonal polynomials, C.R. Acad. Sci. Paris Sér. 1. Math., 307 (1988), pp. 559-564.

[10] P. G. NEVAI AND J. S. DEHESA, On asymptotic average properties of zeros of orthogonal polynomials, SIAM J. Math. Anal., 10 (1979), pp. 1184-1192.

[11] M. RAHMAN, A simple proof of Koornwinder's addition formula for the little q-Legendre polynomials, Proc. Amer. Math. Soc., 107 (1989), pp. 373-381.

[12] M. Rahman AND A. Verma, Product and addition formula for the continuous q-ultraspherical polynomials, SIAM J. Math. Anal., 17 (1986), pp. 1461-1474.

[13] L. L. VAKSMAN AND YA. S. SOIBELMAN, Function algebra on the quantum group SU(2), Funktsional. Anal. i Prilozhen, 22 (1988), pp. 1-14. (In Russian.) Funct. Anal. Appl., 22 (1988), pp. 170-181.

[14] W. Van Assche, Asymptotics for Orthogonal Polynomials, Lecture Notes in Math., 1265, SpringerVerlag, Berlin, New York, 1987.

[15] W. VAN ASSCHE AND J. S. GERONIMO, Asymptotics for orthogonal polynomials with regularly varying recurrence coefficients, Rocky Mountain J. Math., 19 (1989), pp. 39-49.

[16] H. S. WALL, A continued fraction related to some partition formulas of Euler, Amer. Math. Monthly, 48 (1941), pp. 102-108.

[17] S. L. Woronowicz, Compact matrix pseudogroups, Comm. Math. Phys., 111 (1987), pp. 613-665.

[18] - Twisted SU(2) group. An example of a non-commutative differential calculus, Publ. Res. Inst. Math. Sci., 23 (1987), pp. 117-181. 